\title{
Averaging techniques for microstructures with localization bands due to damage progression
}

Despite the complex manufacturing process and the relatively high manufacturing costs involved, the use of carbon fiber reinforced plastics (CFRP) is constantly increasing in lightweight constructions. However, due to their brittle damage behavior, high safety factors are required to prevent catastrophic failure. More insight into the microscopic failure mechanisms is needed. Multiscale analysis of representative volume elements (RVEs) can provide insight into the microscopic material behavior and failure mechanisms.

However, standard first order homogenization schemes based on the so-called Hill condition $^{[1]}$ fail in the presence of localization bands, because a representative size of the RVE cannot be identified ${ }^{[2]}$. In order to overcome this problem, Nguyen et al. ${ }^{[3]}$ have proposed the so-called failure zone averaging scheme, which allows the definition of a representative RVE-size even after localization bands have occurred.

In the current work, this approach is adopted to provide micro-scale simulations of the microscopic failure of CFRPs. Here, the matrix material is equipped with a micromorphic brittle damage model, while cohesive zone elements are used to simulate the interface debonding of carbon fibers and resin matrix. Results using the failure zone averaging for various microstructures and different external loadings will be presented and discussed.

[1] Hill, R. [1972], 'On constitutive macro-variables for heterogeneous solids at finite strain', Proceedings of the Royal Society of London. A. Mathematical and Physical Sciences 326(1565), 131-147.

[2] Gitman, I. M., Askes, H., Sluys, L. J. and Valls, O. L. [2004], The concept of representative volume for elastic, hardening and softening materials, in 'Proceedings of XXXII International Summer School-Conference 'Advance problems in Mechanics", pp. $180-184$.

[3] Nguyen, V. P., Lloberas-Valls, O., Stroeven, M. and Sluys, L. J. [2010], 'On the existence of representative volumes for softening quasi-brittle materials - a failure zone averaging scheme', Computer Methods in Applied Mechanics and Engineering 199(45), $3028-3038$. 